\title{
MHD Peristaltic flow of a nanofluid in a constricted artery for different shapes of nanosized particles
}

https://doi.org/10.1515/nleng-2017-0064

Received May 24, 2017; revised August 26, 2017; accepted December $2,2017$.

\begin{abstract}
MHD peristaltic transport of copper-water nanofluid in an artery with mild stenosis for different shapes of nanoparticles is studied in this paper. The exact solution is obtained for velocity, temperature and pressure gradient. The influence of all the parameters on velocity, temperature and pressure gradient is observed. The effect of stenosis plays a vital role in this paper, as it finds its application in the field of medicine. It is observed that the nanofluid flows rapidly in the presence of stenosis, which helps in destroying of the stencils soon. The shape of the nanoparticle is another important point to be concentrated due to based on the shape of the nanoparticle the fluid flow depends. The shape of the nanoparticle should be considered as bricks to increase the velocity, temperature and the pressure gradient. But a reversed behavior is observed if the shape of the nanoparticle is chosen as platelets. Streamlines are also concentrated and it is observed that there are more number of boluses if the shape of the nanoparticle is chosen as bricks. The effect of volume of the solid nanoparticle and stenosis warrants further study of the flow of nanofluids in tube and channel.
\end{abstract}

Keywords: MHD, peristaltic flow, Artery, Cu-water Nanofluid, Mild stenosis

Palluru Devaki, Department of Mathematics, CMR University, Bangulor, Karnataka, India

*Corresponding Author: Bhumarapu Venkateswarlu, Department of Mathematics, Walchand Institute of Technology, Solapur-413006, M.H., India, E-mail: bvenkateswarlu.maths@gmail.com

Suripddi Srinivas, School of Advanced Sciences, VIT University, Vellore, Tamil Nadu, 632014, India

Sreedharamalle Sreenadh, Department of Mathematics, Sri

Venkateswara University, Tirupati, A.P., India

\section{Introduction}

In recent years significant interest has been developed surrounding the utilization of micro and nanofabrication techniques for the construction novel biomaterials and implants. Developed from advances in the fields of computer science, engineering, physics and biology, these techniques have found widespread application in an array of industries including computing consumer electronics, manufacturing and biotechnology. While the applications of these techniques toward biomaterials in relatively new, they promise to enable the creation of smaller, more active and more dynamic implants and devices. Nanotechnology is one of the most exciting new technologies of the twenty-first century. With its increasingly important and diverse impact on a variety of sectors such as biotechnology, energy, electronics and consumer goods, it promises to transform our lives within this decade. Anghel and Grumezescu [1] demonstrated the characteristics of nanofluid oils in the smart design of novel material surfaces for prosthetic devices. Sheikholeslami and Rokni [2] examined the Buongiorno model for the effect of melting heat transfer of nanofluid flow due to magnetic field. Akbar [3] implemented the biological analysis of nano Prandtl fluid model due to diverging tube. The ciliaryinduced transport in MHD nanofluid flow model was presented by Akbar et al. [4]. Further, many researchers [5-9] have investigated the heat transfer flow of nanofluids considering the different geometries.

Nanoparticles play a major role in nanobiotechnology. In fact, applications involving nanoparticles reagents have already been commercially developed to a high degree. Penn et al. [10] have summarized the use of nanoparticles in biotechnology in an excellent review. Application of drug delivery in MHD peristaltic blood flow of nanofluid in non-uniform channel was investigated by Abbas et al. [11]. Shit et al. [12] presented the MHD flow of Biofluid induced by peristaltic wave. They found that the trapping bolus strongly depends on electro-osmotic parameter and magnetic field strength. Sheikholeslami and Rokni [13] considered the Buongiorno model for the melting heat transfer effects on nanofluid flow due to magnetic field. Abbasi 
et al. [14] analyzed the slip effects on peristaltic transport of Cu-water nanofluid in channel. Peristaltic transport of nanofluid in a compliant wall cannel with thermal radiation and convective conditions was presented by Hayat et al. [15]. Later Hayat et al. [16] examined the mixed connective peristaltic transport of Carreau-Yasuda nanofluid in a tapered asymmetric channel. Sheikholeslami et al. [17] investigated on the nanofluid flow inside a porous cavity considering various shapes of nanoparticles by using Mesoscopic method. Later, Sheikholeslami [18] analyzed the convective nanofluid flows due to magnetic field with shape effect of nanoparticles. Nanoparticles were investigated through a horizontal tube experimentally by Zhaoqin and Zhongping [19]. Mori and Hegmann [20] studied on composition of gold nanoparticles.

The abnormal and unnatural growth in the lumen of an artery is called stenosis. The arteries are narrowed by the development of atherosclerotic plaques that protrude into the lumen, resulting arterial stenosis. This led to extensive investigations of the characteristics of blood flow through a stenosed artery by [21-25]. Herschel Bulkley fluid was modeled by Sanjeev et al. [26] through an arterial segment with stenosis. Peristaltic flow of a Jeffrey nanofluid in a tapered artery with mild stenosis and slip condition was investigated by Nabil et al. [27]. Nadeem and Ijaz [28] investigated the metallic nanoparticles on blood flow through stenosed artery with permeable walls. Ferromagnetic effects for nanofluid venture through composite permeable stenosed arteries were analyzed by Akbar and Mustafa [29]. Akbar [30] displayed the Ferromagnetic CNT suspended $\mathrm{H}_{2} \mathrm{O}+\mathrm{Cu}$ nanofluid analysis through composite stenosed arteries with permeable wall. Mekheimer et al. [31] investigated the metallic nanoparticles on blood flow through a stenotic artery. Nabil et al. [32] analyzed the peristaltic flow of a sisko fluid with mild stenosis through a porous medium with slip effects. Ahmed and Nadeem [33] examined the different nanoparticles as antimicrobials of blood flow through diseased arteries. Recently, Srinivasacharya and Madhava Rao [34] studied the modeling of blood flow through a bifurcated artery using nanofluid.

Motivated by above discussed papers in the field of nanoparticles and stenoses, we studied on the MHD peristaltic flow of a Cu-Water nanofluid in an artery with mild stenosis for different shapes of nanoparticles. The governing equations are solved analytically by using a long wave length assumption. The exact solution is obtained for velocity, temperature and the pressure gradient. The effect of different parameters on velocity, temperature and the pressure gradient is investigated through graphs.

\section{Mathematical formulation of the problem}

Consider the peristaltic flow of a copper water nanofluid in an axisymmetric tube with mild stenosis is of finite length $L+d$. The tube wall undergoes a peristaltic movement. The axisymmetric nature of the tube makes us to choose cylindrical coordinate system $(r, \phi, z)$, where $r$ denotes the radial coordinate, $\phi$ denotes the azimuthal angle and $z$ denotes the axial coordinate. The stenosis is developed in an axially symmetric manner. The radius of the tube is assumes as a function of $z$ according to Sanjeev [26] it is given by

$R(z)= \begin{cases}R_{1}-m \delta(z+L) & L<z<-z_{0} \\ R_{1}-m \delta(z+L)-\frac{H}{2}\left(1+\operatorname{Cos} \frac{z \pi}{z_{0}}\right) & -z_{0} \leq z \leq z_{0} \\ R_{1}-m \delta(z+L) & z_{0}<z<d\end{cases}$

Here $R(z)$ represents the radius of the tapered artery, $R_{1}$ represents the radius of the un-tapered artery, $\delta=\frac{R_{1}}{\lambda}, \lambda$ is the wave length, $H=h \cos \theta$ is the height of the stenosis in the tapered artery, $\theta$ is the angle of the tapering, $h$ is the maximum height of the stenosis, $z_{0}$ is the half length of the stenosis and $m=\operatorname{Tan} \theta$ is the slope of the tapered vessel.

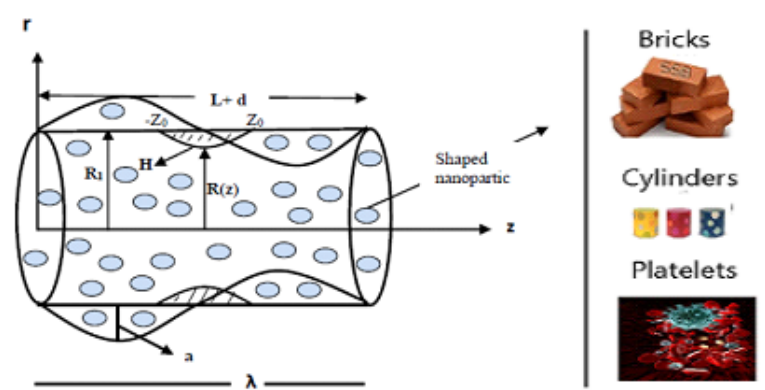

Fig. 1: Physical model of the problem

The peristaltic wave propagating the tube wall is given by

$$
h=a \operatorname{Cos} \frac{2 \pi}{\lambda}\left(\bar{Z}-\frac{k \bar{t}}{R_{1}}\right)
$$

where $a$ is the wave speed, $\lambda$ is the wave length and $c=\frac{k}{R_{1}}$ is the wave speed

The transformations between fixed and wave frames are

$\bar{r}=\bar{R} \quad \bar{z}=\bar{Z}-c \bar{t} \quad \bar{v}=\bar{V} \quad \bar{w}=\bar{W}-c \quad \bar{p}(\bar{z}, \bar{r}, \bar{t})=\bar{p}(\bar{Z}, \bar{R}, \bar{t})$

The equations governing the flow of a nanofluid in a circular tube is given by

$$
\frac{1}{r} \frac{\partial(r u)}{\partial r}+\frac{\partial w}{\partial z}=0
$$




$$
\begin{gathered}
u \frac{\partial u}{\partial r}+w \frac{\partial u}{\partial z}=-\frac{1}{\rho_{n f}} \frac{\partial p}{\partial r}+\frac{\mu_{n f}}{\rho_{n f}} \frac{\partial}{\partial r}\left\{2 \frac{\partial u}{\partial r}\right\} \\
+\frac{\mu_{n f}}{\rho_{n f}} \frac{2}{r}\left\{\frac{\partial u}{\partial r}-\frac{u}{r}\right\}+\frac{\mu_{n f}}{\rho_{n f}} \frac{\partial}{\partial z}\left\{\frac{\partial u}{\partial r}+\frac{\partial w}{\partial z}\right\} \\
u \frac{\partial u}{\partial r}+w \frac{\partial u}{\partial z}=-\frac{1}{\rho_{n f}} \frac{\partial p}{\partial z}+\frac{\mu_{n f}}{\rho_{n f}} \frac{\partial}{\partial z}\left\{2 \frac{\partial w}{\partial z}\right\}+\frac{\mu_{n f}}{\rho_{n f}} \frac{2}{r}\left\{\frac{\partial u}{\partial r}-\frac{u}{r}\right\} \\
+\frac{\mu_{n f}}{\rho_{n f}} \frac{1}{r} \frac{\partial}{\partial r}\left\{r\left(\frac{\partial u}{\partial z}+\frac{\partial w}{\partial r}\right)\right\}-\frac{\sigma B_{0}^{2}}{\rho_{n f}}\left(w+\frac{k}{R_{1}}\right)+g \alpha\left(T-T_{0}\right) \\
u \frac{\partial T}{\partial r}+w \frac{\partial T}{\partial z}=\frac{k_{n f}}{\left(\rho_{c p}\right)_{n f}}\left\{\frac{\partial^{2} T}{\partial r^{2}}+\frac{1}{r} \frac{\partial T}{\partial r}+\frac{\partial^{2} T}{\partial z^{2}}\right\}+\frac{Q_{0}}{\left(\rho_{c p}\right)_{n f}}
\end{gathered}
$$$$
u \frac{\partial u}{\partial r}+w \frac{\partial u}{\partial z}=-\frac{1}{\rho_{n f}} \frac{\partial p}{\partial z}+\frac{\mu_{n f}}{\rho_{n f}} \frac{\partial}{\partial z}\left\{2 \frac{\partial w}{\partial z}\right\}+\frac{\mu_{n f}}{\rho_{n f}} \frac{2}{r}\left\{\frac{\partial u}{\partial r}-\frac{u}{r}\right\} \quad \frac{d p}{d z}=\frac{1}{(1-\phi)^{2.5}} \frac{1}{r} \frac{\partial}{\partial r}\left(r \frac{\partial w}{\partial r}\right)-M^{2}(w+1)+G_{r} \theta
$$

non-dimensional governing equations become (dropping dashes)

$$
\frac{\partial p}{\partial r}=0
$$

$$
\frac{1}{r} \frac{\partial}{\partial r}\left(r \frac{\partial \theta}{\partial r}\right)+\xi\left\{\frac{k_{s}+(\omega+1) k_{f}+\left(k_{f}-k_{s}\right) \phi}{k_{s}+(\omega+1) k_{f}-(\omega+1)\left(k_{f}-k_{s}\right) \phi}\right\}=0
$$

Where $M$ is the Hartmann number, $\xi$ is the heat absorption parameter and $G_{r}$ is the Grashof number

The corresponding dimensionless boundary conditions are

$$
\begin{aligned}
& \frac{\partial w}{\partial r}=0 \frac{\partial \theta}{\partial r}=0 \text { at } \quad r=0 \\
& w=-1 \theta=0 \text { at } r=h(z)+R(z)
\end{aligned}
$$

by $T$. Effective density is given by $\rho_{n f}$, effective dynamic viscosity is denoted by $\mu_{n f}$, heat capacitance is denoted by $\left(\rho_{c p}\right)_{n f}$, effective thermal diffusitivity is denoted by $\alpha$, and effective thermal conductivity of the nanofluid is denoted by $k_{n f}$, which are defined as (Akbar and Butt [35])

$$
\begin{aligned}
& \rho_{n f}=(1-\phi) \rho_{f}+\phi \rho_{f} \mu_{n f}=\frac{\mu_{f}}{(1-\phi)^{2.5}} \\
& \left(\rho_{c p}\right)_{n f}=(1-\phi)\left(\rho_{c p}\right)_{f}+\phi\left(\rho_{c p}\right)_{s}
\end{aligned}
$$

Where the solid nanoparticle volume fraction is $\phi$ and the thermal conductivity is given by

$$
k_{n f}=k_{f}\left(\frac{k_{s}+(\omega+1) k_{f}-(\omega+1)\left(k_{f}-k_{s}\right) \phi}{k_{s}+(\omega+1) k_{f}+\left(k_{f}-k_{s}\right) \phi}\right)
$$

Where $k_{f}$ and $k_{s}$ are the conductivities of the particle material and the base fluid, $\omega$ is the shape factor. According to Akbar and Butt [35], the shape factor has different values which are given in the following table

Table 1: Nanoparticle shape and shape factor

\begin{tabular}{ll}
\hline Nanoparticle Shape type & Shape Factor \\
\hline Bricks & 3.7 \\
Cylinders & 4.9 \\
Platelets & 5.7 \\
\hline
\end{tabular}

The non-dimensional quantities are given by

$$
\begin{aligned}
& \bar{r}=\frac{r}{R_{1}} \bar{z}=\frac{z}{\lambda} \bar{u}=\frac{u R_{1}}{k \delta} \quad \bar{w}=\frac{w R_{1}}{k} \overline{h_{1}}=\frac{h_{1}}{R_{1}} \\
& \bar{p}=\frac{p R_{1}{ }^{3}}{\mu_{f} \lambda k} \bar{t}=\frac{k t}{R_{1} \lambda} \quad \overline{z_{0}}=\frac{z_{0}}{\lambda} \quad \bar{L}=\frac{L}{\lambda}
\end{aligned}
$$

Using (8) in equations (3-6) and making use of the assumption of long wave length approximation, the
Where $h(z)=1+\varepsilon \operatorname{Cos}(2 \pi z)$ and $R(z)=$
$\left\{\begin{array}{l}1-m(z+L) L<z<-z_{0} \\ 1-m(z+L)-\frac{H}{2}\left\{1+\operatorname{Cos} \frac{z \pi}{z_{0}}\right\}-z_{0} \leq z \leq z_{0} \\ 1-m(z+L) z_{0}<z<d\end{array}\right.$

\section{Exact solution of the problem}

Solving equations (9), (10) \& (11) using the boundary conditions (12) \& (12), we obtain

$$
\begin{aligned}
& w(r, z)=\frac{1}{4 M^{4} \sqrt{(1-\phi)^{2.5}}} \\
& \left\{\begin{array}{c}
\frac{4 I_{0}\left\{r M \sqrt{(1-\phi)^{2.5}}\right\}\left\{G_{r} \frac{k_{f}}{k_{n n}} \xi+M^{2} \frac{d p}{d z}(1-\phi)^{2.5}\right\}}{I_{0}\left\{(h+R) M \sqrt{(1-\phi)^{2.5}}\right\}}-4 M^{2}(1-\phi)^{2.5} \\
\left\{M^{2}+\frac{d p}{d z}\right\}+G_{r} \xi \frac{k_{f}}{k_{n f}}\left\{M^{2}\left((h+R)^{2}-r^{2}\right)(1-\phi)^{2.5}-4\right\}
\end{array}\right\}
\end{aligned}
$$

$$
\theta(r, z)=\frac{\xi\left((h+R)^{2}-r^{2}\right)}{4}\left\{\frac{k_{s}+(\omega+1) k_{f}+\left(k_{f}-k_{s}\right) \phi}{k_{s}+(\omega+1) k_{f}-(\omega+1)\left(k_{f}-k_{s}\right) \phi}\right\}
$$

The flow rate is given by

$$
F=2 \int_{0}^{h+R} r w d r
$$


From this we can find the pressure gradient as

$$
\begin{aligned}
& \frac{d p}{d z}=\left[M \sqrt{(1-\phi)^{2.5}} I_{0}\left\{M(h+R) \sqrt{(1-\phi)^{2.5}}\right\}\right. \\
& \left\{G_{r}(h+R)^{2} \frac{k_{f}}{k_{n f}} \xi\left((h+R)^{2} M^{2}(1-\phi)^{2.5}-8\right)-8 M^{4}\left(F+(h+R)^{2}\right)(1-\phi)^{2.5}\right\} \\
& \left.+16 G_{r}(h+R) \frac{k_{f}}{k_{n f}} \xi I_{1}\left\{(h+R) M \sqrt{(1-\phi)^{2.5}}\right\}\right] /\left[8 M^{3}(h+R)^{2} \sqrt{(1-\phi)^{7.5}} I_{2}\left\{(h+R) M \sqrt{(1-\phi)^{2.5}}\right\}\right]
\end{aligned}
$$

\section{Results and discussions}

The Peristaltic flow of copper water Nanofluid in an artery with mild stenosis is investigated along with the effect of MHD. The effect of different parameter is discussed in the form of graphs in Figures 2-7. The graphical representation is done with the help of MATLAB software. The velocity, temperature and pressure gradient are obtained by exact solution approach. In the absence of stenosis the results agree with the results of Akbar \& Butt [35].

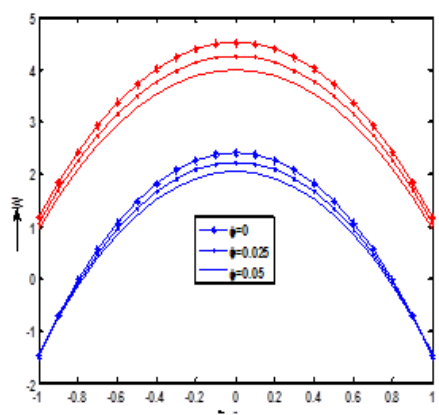

(a)

Fig. 2: (a) Velocity profile for various values of $\phi$ (b) Temperature profile for various values of $\phi$ (c) Pressure gradient for various values of $\phi$

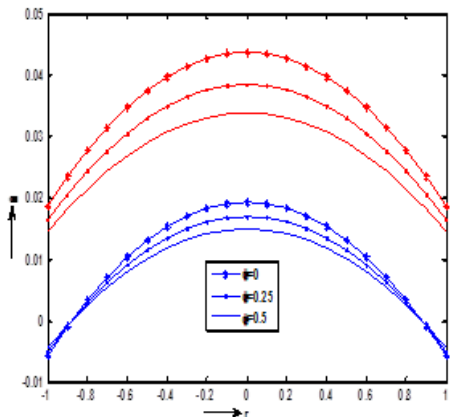

(b)

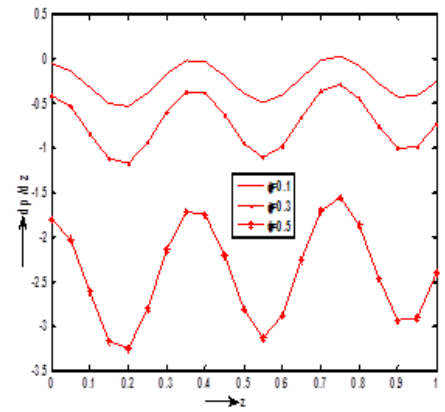

(c) (a)

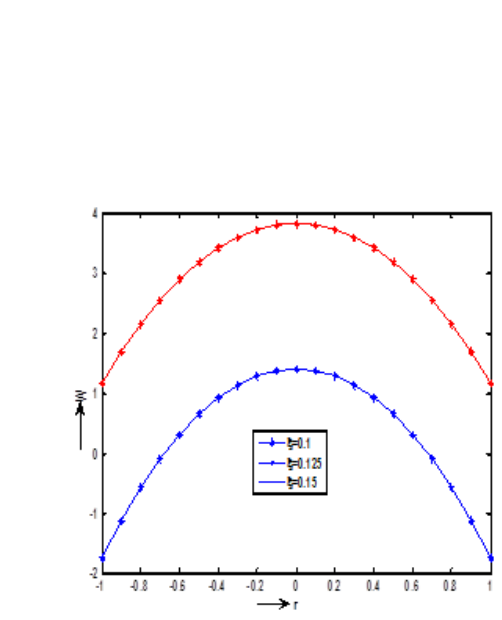

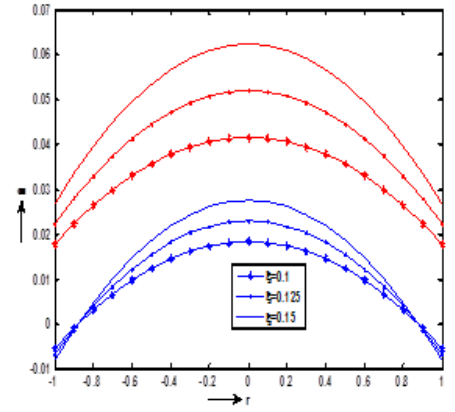

(b)

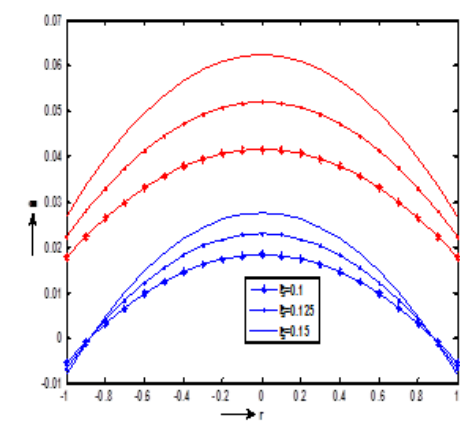

(c)

Fig. 3: (a) Velocity profile for various values of $\xi$ (b) Temperature profile for various values of $\xi$ (c) Pressure gradient for various values of $\xi$ 


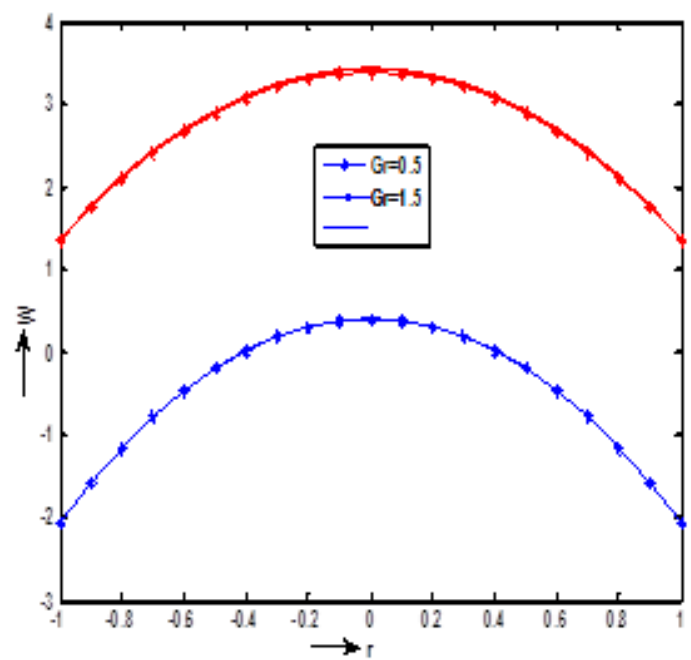

(a)

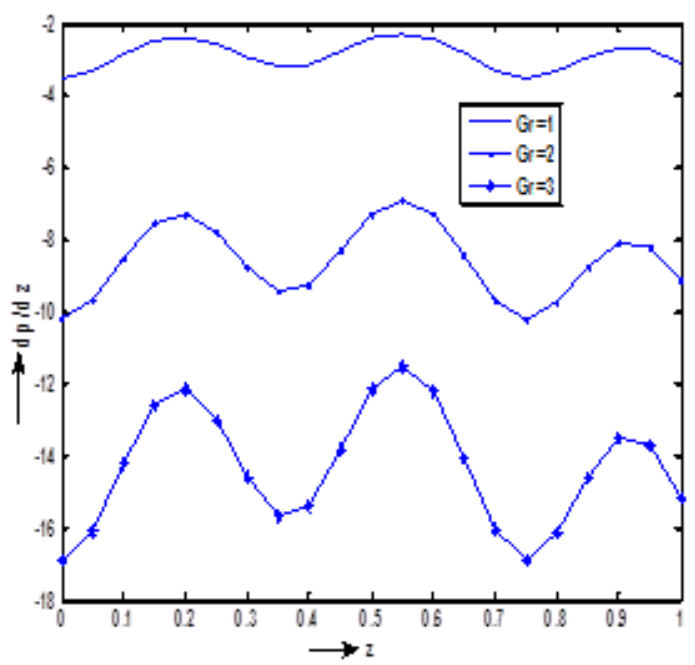

(b)

Fig. 4: (a) Velocity profile for various values of $\mathrm{Gr}$ (b) Pressure gradient for various values of $\mathrm{Gr}$

Figures 2(a) to 2(c) display the fluid velocity, temperature and pressure gradient for various values of the volume of solid nanoparticle $\phi$ with two different situations (i.e. with stenosis and without stenosis). It is observed that as the volume of the solid nanoparticle increases the fluid velocity and temperature is decreasing in both the presence and in the absence of stenosis. This means that in the presences of stenosis the nanofluid flow is rapid, when compared with the flow in the absence of stenosis. Also, the pressure gradient decreases with increasing values of $\phi$.

The variation of fluid velocity, temperature and pressure gradient for the effect of heat absorption parameter $\xi$

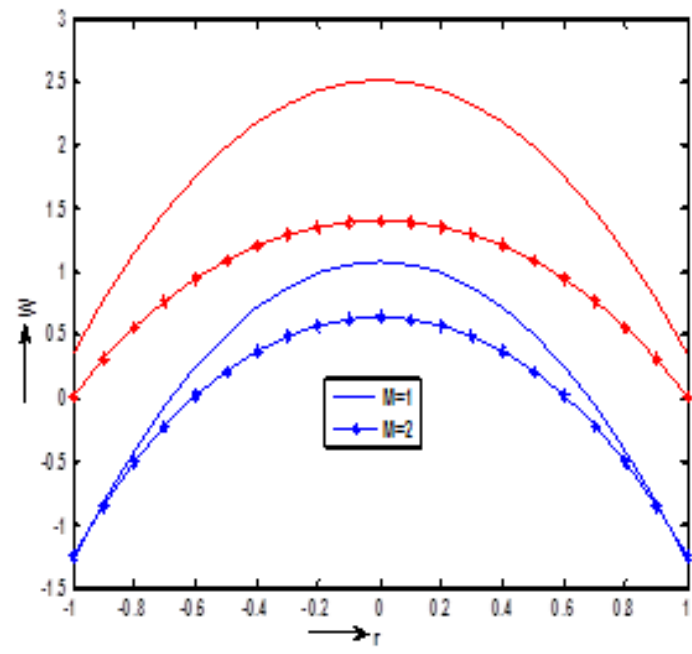

(a)

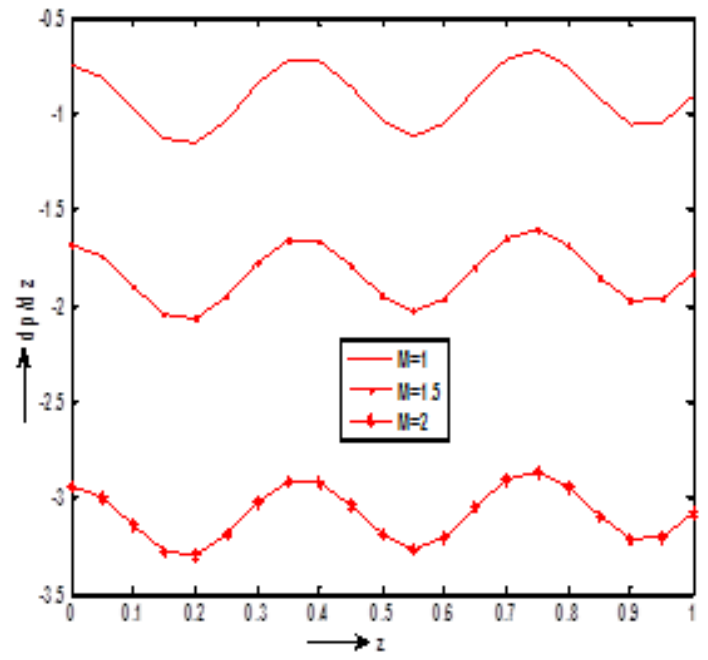

(b)

Fig. 5: (a) Velocity profile for various values of $M$ (b) Pressure gradient for various values of $M$

with and without stenosis is shown in Figures 3(a)-3(c). It is clear that the fluid velocity and temperature increasing both in the presence and absence of stenosis with increasing values of $\xi$ whereas the reverse effect occurring in the pressure gradient. Further, it is found that the velocity and temperature is high in the presence of stenosis rather than in the absence of stenosis.

Figures 4(a) \& 4(b) elucidate the variation of velocity and pressure gradient with different values of Grashof number $G r$ in both cases regarding with stenosis and without stenosis. It is clear that the velocity of diminishing by the improving values of $G r$ whereas the opposite behavior is observed with $\mathrm{Gr}$. That is as the Grashof number in- 


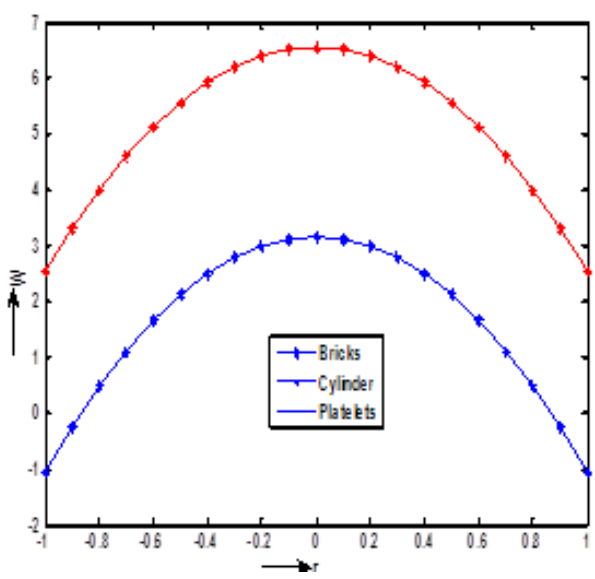

(a)

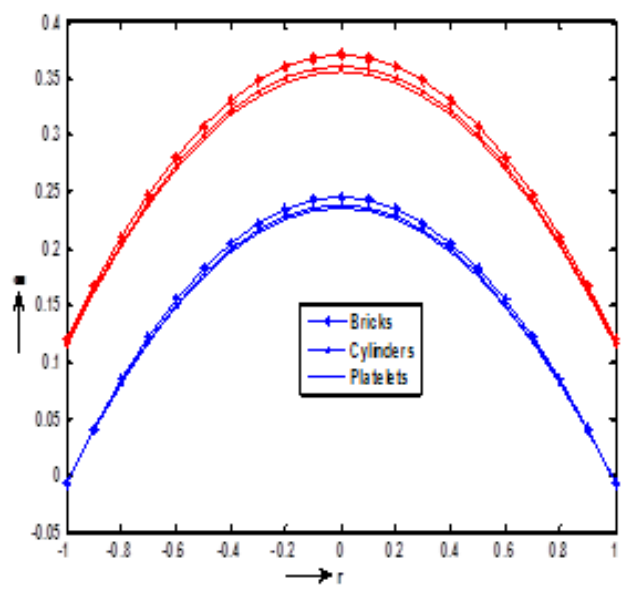

(b)

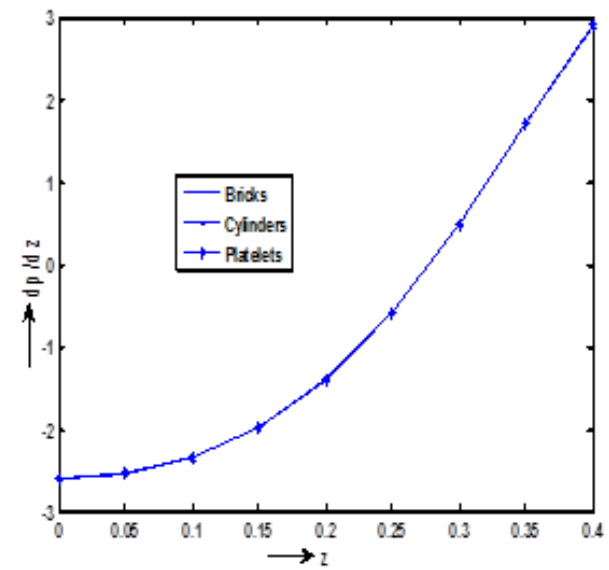

(c)

Fig. 6: (a) Velocity profile for various values of shape factor (b) Temperature profile for various values of shape factor (c) Pressure gradient for various values of shape factor

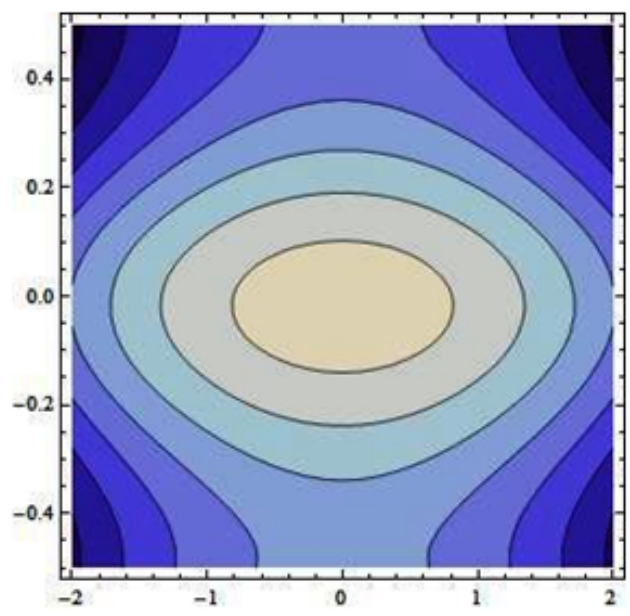

(a) Bricks

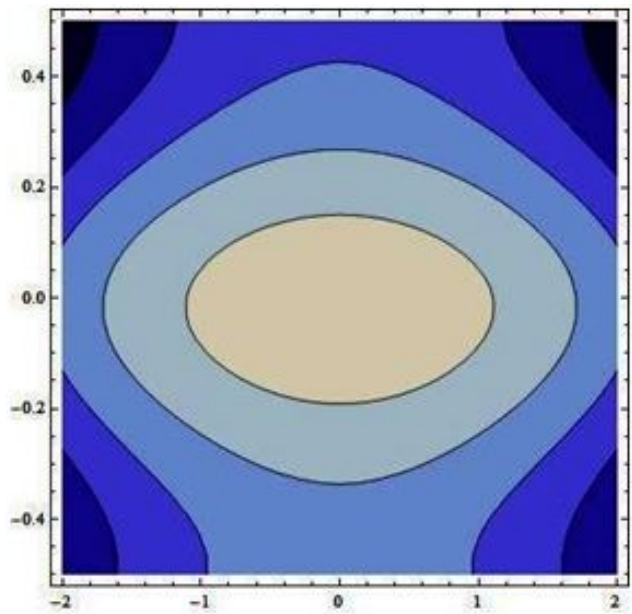

(b) Cylindrical

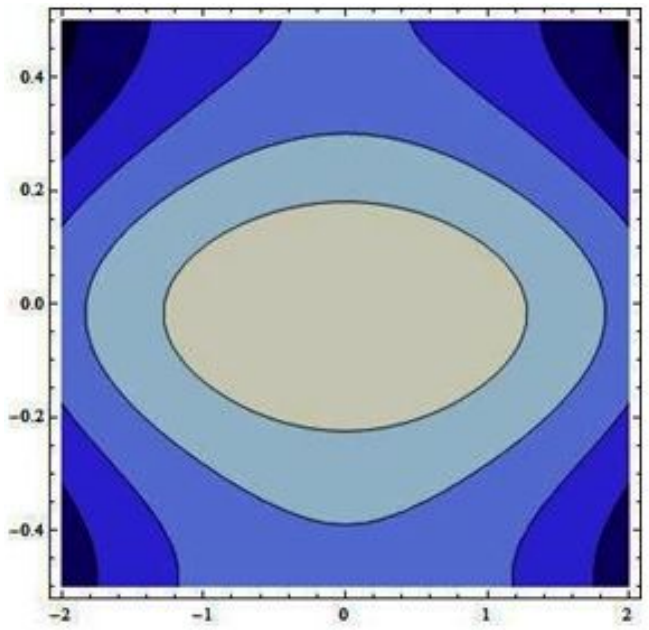

(c) Platelets

Fig. 7: Effect of various shapes of the shape factor 
creases the pressure gradient is increasing in the presence of stenosis, which is depicted in Figure 4(b).

The velocity profile and pressure gradient are shown in Figures 5(a) \& 5(b) with radius for different volumes of Hartmann number $M$. It is clear that the fluid velocity decreasing in the presence of stenosis and absence of stenosis for rising values of Hartmann number. Also, it is observed that the pressure gradient decreases with increasing values of $M$.

The investigation of the fluid velocity, temperature profiles and pressure gradient in the presence of stenosis and in the absence of stenosis for different shapes of the nanoparticles is depicted in Figure 6(a) to 6(c). It is noticed that the velocity and temperature is more, if the nanoparticles shape is chosen as bricks rather than choosing nanoparticles shape as cylinders and platelets. Further, the pressure gradient is more when the shape of the nanoparticle is in the form of bricks and therefore this effect is similar both in the presence and in the absence of stenosis.

\subsection{Trapping}

The trapping phenomenon is an important factor to be considered in peristaltic motion of fluids. In a wavy frame some streamlines are separated to form a closed region called trapped bolus under specific conditions. This trapped bolus is moved as a whole with the speed of the peristaltic wave. The stream line pattern in a circular tube for different shapes of nanoparticles is shown in Figure $7(\mathrm{a}, \mathrm{b}, \mathrm{c})$. It is observed that the number of the boluses is decreasing. In the presence of stenosis if the shape of the nanoparticle is selected as bricks three boluses were observed and only two boluses are formed if the shape of the nanoparticle is chosen as cylinder and platelets. Also it noticed that the size of the bolus decreases if the shape of the nanoparticle is preferred as platelets.

\section{Conclusions}

Nanofluid flow in an artery with mild stenosis is studied under the effect of peristalsis. The Nano fluid is considered as a combination of copper and water. Observe the resluts are

1. The nanofluid flow is more in the presence of stenosis.

2. As the Nanoparticle size increases the fluid flow and the temperature decreases.
3. As the amplitude ratio of the peristalsis increases the temperature is increasing.

4. If the shape of the nanoparticle is selected as bricks, then the temperature of the fluid flow increases and the pressure gradient will decrease.

5. Pressure gradient decreases as the Grashof number and heat absorption parameter increases.

6. In the absence of stenosis the pressure gradient has insignificant change for different shapes of nano particles.

7. Shape of the nanoparticle has to be elected as bricks for more number of boluses.

\section{Nomenclature}

$a$ wave speed

$c$ wave speed

$L+d$ length of the tube

$\mathrm{Gr}$ Grashof number

$H$ height of the stenosis in the tapered artery

$h$ maximum height of the stenosis

$k_{f}$ conductivities of the particle base fluid

$k_{s}$ conductivities of the particle material

$k_{n f}$ thermal conductivity of the nanofluid

$M$ Hartmann number

$\mathrm{nm}$ slope of the tapered vessel

$r$ radial coordinate

$R$ radius of the tapered artery

$R_{1}$ radius of the un-tapered artery

$T$ local temperature of the fluid

$(u, w)$ velocity components in $r$ and $z$ directions

$z$ axial coordinate

$z_{0}$ half length of the stenosis

\section{Greek Symbols}

$\alpha$ thermal diffusitivity

$\phi$ azimuthal angle

$\theta$ angle of the tapering

$\lambda$ wave length

$\xi$ heat absorption parameter

$\omega$ shape factor

$\rho_{n f}$ density

$\mu_{n f}$ dynamic viscosity

$\left(\rho_{c p}\right)_{n f}$ heat capacitance 


\section{References}

[1] I. Anghel, and A.M. Grumezescu, Hybrid nanostructured coating for increased resistance of prosthetic devices to staphylococcal colonization. Nanoscale research letters, 8, 2013.

[2] M. Sheikholeslami, Numerical simulation of magnetic nanofluid natural convection in porous media. Physics Letters A, 381, 2017: 494-503.

[3] N.S. Akbar, Biological analysis of nano Prandtl fluid model in a diverging tube. Journal of Computational an Theoretical Nanoscience, 12, 2015: 1-8.

[4] N.S. Akbar, D. Tripathi, Z.H. Khan and O.A. Beg, Mathematical model for ciliary-induced transport in $\mathrm{MHD}$ flow of $\mathrm{Cu}-\mathrm{H}_{2} \mathrm{O}$ nanofluid with magnetic induction. Chinese Journal of Physics, 2017.

[5] P.V. Satya Narayana and B. Venkateswarlu, Heat and mass transfer on MHD nanofluid flow past a vertical porous plate in a rotating system. Frontiers in Heat and Mass Transfer, 7 (8), 2016: 1-10.

[6] B. Venkateswarlu and P.V. Satya Narayana, Chemical reaction and radiation absorption effects on the flow and heat transfer of a nanofluid in a rotating system. Applied Nanoscience, 5, 2015: 351-360.

[7] P. V. Satya Narayana, B. Venkateswarlu and S. Venkataramana, Thermal radiation and heat source effects on a MHD nanofluid past a vertical plate in a rotating system with porous medium. Journal of Heat Transfer Asian Research, 44(1), 2015: 1-19.

[8] M. Sheikholeslami and H.B. Rokni, Effect of melting heat transfer on nanofluid flow in existence of magnetic field considering Buongiorno Model. Chinese Journal of Physics, 55, 2017, 1115-1126.

[9] M.K. Nayak, MHD 3D flow and heat transfer analysis of nanofluid by shrinking surface inspired by thermal radiation and viscous dissipation. International Journal of Mechanical Sciences, 124 (125), 2017: 185-193.

[10] S.G. Penn, L. He and M.J. Natan Nanoparticles in Bioanalysis, Current Opinion in Chemical Biology, 7(5), 2003: 609-615.

[11] M.A. Abbas, Y.Q. Bai, M.M. Rashidi and M.M. Bhatti, Application of drug delivery in Magnetohydrodynamics peristaltic blood flow of nanofluid in a non-uniform channel. J. Mech. Med. Biol., 16, 2015:1650-052

[12] G.C. Shit, N.K. Ranjit A. Sinha, Electro-magnetohydrodynamic Flow of Biofluid Induced by Peristaltic Wave: A Non-Newtonian Model. Journal of Bionic Engineering, 13(3), 2016: 436-448.

[13] M. Sheikholeslami and H.B. Rokni, Effect of melting heat transfer on nanofluid flow in existence of magnetic field considering Buongiorno Model. Chinese Journal of Physics, 55 2017: 1115-1126.

[14] F.M. Abbasi, T. Hayat, B. Ahmad and G.Q. Chen, Slip effects on mixed convective peristaltic transport of copper-water nanofluid in an inclined channel. Plos one, 9(8), 2014.

[15] T. Hayat, Z. Nisar, H. Yasmin and A. Alsaedi, Peristaltic transport of nanofluid in a compliant wall channel with convective conditions and thermal radiation. Journal of Molecular Liquids, 220, 2016: 448-453

[16] T. Hayat, R. Iqbal, A. Tanveer and A. Alsaedi, Mixed convective peristaltic transport of Carreau-Yasuda nanofluid in a tapered asymmetric channel. Journal of Molecular Liquids, 223, 2016: 1100-1113.
[17] M. Sheikholeslami, Mohammadkazem Sadoughi, Mesoscopic method for MHD nanofluid flow inside a porous cavity considering various shapes of nanoparticles. International Journal of Heat and Mass Transfer, 113, 2017: 106-114.

[18] M. Sheikholeslami, M. M. Bhatti, Forced convection of nanofluid in presence of constant magnetic field considering shape effects of nanoparticles. International Journal of Heat and Mass Transfer, 111, 2017: 1039-1049

[19] Y. Zhaoqin and D. Zhongping, Investigating the nanoparticles penetration efficiency through horizontal tubes using an experimental approach. Adv. Math. Phy., 2015: 1-6.

[20] T. Mori and T. Hegmann, Determining the composition of gold nanoparticles: a compilation of shapes, sizes, and calculations using geometric considerations. Journal of Nanopar. Res., 2016:18-295.

[21] D.F. Young, Effects of time dependent stenosis of flow through a tube. Journal of Eng. Ind. Trans. ASME, 90, 1968: 248.

[22] S. Wada and T. Karino, Computational studies on LDL transfers from flowing blood to arterial walls. In Clinical Application of Computational Mechanics to the Cardiovascular System. Springer Tokyo, Japan, 2000: 157-173.

[23] J.C. Misra, A. Sinha G.C. Shit, Theoretical analysis of blood flow through an arterial segment having multiple stenosis. J. Mech. Med. Biol., 8, 2008: 265-279.

[24] N.S. Akbar, T. Hayat, S. Nadeem, A. Hendi and Awatif, Influence of mixed convection on blood flow of Jeffrey fluid through a tapered stenosed artery. Thermal Science, 2013, 17: 533546.

[25] R.S. Harjeet Kumar, Chandel, Sanjeev Kumar and Sanjeet Kumar, Non-Newtonian arterial blood flow model through multiple stenosis. Inter. J. Lat. Res. Sci. Tech., 2, 2014: 116-121.

[26] Sanjeev Kumar, N.R. Garg, and Agraj Gupta, Herschel Bulkley model for blood flow through an artieral segment with stenosis. Inter. J. Sci. Tech. Manag., 4, 2015: 93-100.

[27] N.T.M. El-dabe, G.M. Hassan, M.A. Mohamed and D.R. Mostapha, Analytical solution of the peristaltic flow of a Jeffrey nanofluid in a tapered artery with mild stenosis and slip condition. Inter. J. Inno. App. Stud., 12, 2015: 1-32.

[28] S. Nadeem and S. Ijaz, Theoretical analysis of metallic nanoparticles on blood flow through stenosed artery with permeable walls. Physics Letters A, 379(6), 2015: 542-554.

[29] N.S. Akbar and M.T. Mustafa, Ferromagnetic effects for nanofluid venture through composite permeable stenosed arteries with different nanosized particles. AlP Advances, 5, 2015:77-102.

[30] N. S. Akbar, Ferromagnetic CNT suspended $\mathrm{H}_{2} \mathrm{O}+\mathrm{Cu}$ nanofluid analysis through composite stenosed arteries with permeable wall. Physica E: Low-dimensional Systems and Nanostructures, 72, 2015: 70-76.

[31] S. Mekheimer, M. Khaled, S. Mohamed and T. Elnaqeeb, Metallic nanoparticles influence on blood flow through a stenotic artery. Inter. J. Pure App. Maths, 107, 2016: 201- 220.

[32] N.T.M. El-dade, G. M. Moatimid, M.A. Hassan and D.R. Mostapha, Effect of partial slip on peristaltic flow of a sisko fluid with stenosis through a porous medium. App. Maths Inf. Sci., 10(2), 2016: 673-687.

[33] A. Ahmed and S. Nadeem, The study of $\left(\mathrm{Cu}, \mathrm{TiO}_{2}, \mathrm{Al}_{2} \mathrm{O}_{3}\right)$ nanoparticles as antimicrobials of blood flow through diseased arteries. Journal of Molecular Liquids, 216, 2016: 615623. 
[34] D. Srinivasacharya \& G. Madhava Rao, Modeling of Blood Flow through a Bifurcated Artery Using Nanofluid. Bio Nano Sci. 7, 2017:464-474.

[35] N.S. Akber and A.W. Butt, Ferromagnetic effects for peristaltic flow of $\mathrm{Cu}$-water nanofluid for different shapes of nanosize particles. App. Nanosci., 6, 2016: 379-385. 